\title{
Effect of achieved hemoglobin level on renal outcome in non-dialysis chronic kidney disease (CKD) patients receiving epoetin beta pegol: MIRcerA CLinical Evidence on Renal Survival in CKD patients with renal anemia (MIRACLE-CKD Study)
}

\author{
Terumasa Hayashi ${ }^{1} \cdot$ Yukari Uemura ${ }^{2} \cdot$ Michiko Kumagai $^{3}\left[\right.$ Masashi Kimpara ${ }^{3} \cdot$ Hiroyuki Kanno $^{3} \cdot$ Yasuo Ohashi $^{4}$. \\ MIRACLE-CKD Study Group
}

Received: 5 March 2018 / Accepted: 24 September 2018 / Published online: 5 October 2018

(c) The Author(s) 2018

\begin{abstract}
Background Previous randomized-controlled trials have shown that targeting higher hemoglobin $(\mathrm{Hb})$ levels using high dose of ESA in non-dialysis chronic kidney disease (NDCKD) patients resulted in poorer cardiovascular outcome; however, it remains unknown how high $\mathrm{Hb}$ levels achieved by ESA in clinical practice dose could affect renal outcome.

Methods In a multicenter prospective observational study, Japanese NDCKD patients with an estimated glomerular filtration rate (eGFR) of $\geq 6 \mathrm{~mL} / \mathrm{min} / 1.73 \mathrm{~m}^{2}$ and renal anemia $(\mathrm{Hb}<11 \mathrm{~g} / \mathrm{dL}$ ) treated with epoetin beta pegol (C.E.R.A.) for the first time were divided into two groups by $\mathrm{Hb}$ level $(<11 \mathrm{~g} / \mathrm{dL}$ or $\geq 11 \mathrm{~g} / \mathrm{dL})$ in Week 12 of C.E.R.A. treatment (Week 12 $\mathrm{Hb})$. Renal outcome was defined as time until the first occurrence of one of the following: progression to renal replacement therapy, serum creatinine doubling, or eGFR falling below $6 \mathrm{~mL} / \mathrm{min} / 1.73 \mathrm{~m}^{2}$. The effect of Week $12 \mathrm{Hb}$ on the onset of renal events was assessed by the Kaplan-Meier and multivariate Cox regression analyses.

Results In the landmark analysis which included 2851 patients, Kaplan-Meier renal survival rate was $37.57 \%$ in the $<11 \mathrm{~g} /$ $\mathrm{dL}$ group and was significantly higher $(51.47 \%)$ in the $\geq 11 \mathrm{~g} / \mathrm{dL}$ group $(P<0.0001)$. Multivariate Cox regression analysis revealed significantly higher risk of renal events in the $<11 \mathrm{~g} / \mathrm{dL}$ group than in the $\geq 11 \mathrm{~g} / \mathrm{dL}$ group (hazard ratio: $1.26 ; 95 \%$ confidence interval: $1.05-1.51 ; P=0.0103$ ).

Conclusions The results suggest that week $12 \mathrm{Hb}$ levels $\geq 11 \mathrm{~g} / \mathrm{dL}$ achieved with C.E.R.A. treatment were associated with better renal outcomes than $\mathrm{Hb}$ levels $<11 \mathrm{~g} / \mathrm{dL}$.
\end{abstract}

Keywords Anemia $\cdot$ Chronic kidney disease $\cdot$ Erythropoiesis-stimulating agents $\cdot$ Renal survival

Electronic supplementary material The online version of this article (https://doi.org/10.1007/s10157-018-1649-0) contains supplementary material, which is available to authorized users.

Michiko Kumagai

ishikawamck@chugai-pharm.co.jp

1 Department of Kidney Disease and Hypertension, Osaka General Medical Center, 3-1-56 Bandai-higashi, Sumiyoshi-ku, Osaka 558-8558, Japan

2 Biostatistics Department, Central Coordinating Unit, Clinical Research Support Center, The University of Tokyo Hospital, Tokyo, Japan

3 Pharmacovigilance Department, Chugai Pharmaceutical Co., Ltd., 2-1-1 Nihonbashi-Muromachi, Chuo-ku, Tokyo 103-8324, Japan

4 Department of Integrated Science and Engineering for Sustainable Society, Chuo University, Tokyo, Japan

\section{Introduction}

Anemia occurs at a relatively early stage in non-dialysis chronic kidney disease (NDCKD) patients [1], and is related to renal function outcome, cardiovascular comorbidities, and mortality [2]. Investigations of the effects of treating anemia with erythropoiesis-stimulating agents (ESAs) have had conflicting findings in terms of renal protection, inhibition of cardiovascular events, and improvement of life expectancy [3-8]. In addition, there have been no conclusions on the optimal hemoglobin $(\mathrm{Hb})$ level for starting ESA therapy or the optimal target $\mathrm{Hb}$ level for maintenance ESA therapy. Gouva et al. compared a group with $\mathrm{Hb}$ maintained at $13 \mathrm{~g} / \mathrm{dL}$ by starting ESA early and a group in which ESA therapy was started at $\mathrm{Hb} \leq 9 \mathrm{~g} / \mathrm{dL}$; they reported a higher renal survival rate when 
ESA therapy was started early [3]. The JET-STREAM study conducted in Japan also found that the early initiation of anemia treatment when $\mathrm{Hb}$ levels decreased below $11 \mathrm{~g} / \mathrm{dL}$ could be more effective at reducing the risk of renal events in NDCKD patients with anemia compared with the initiation of ESA therapy at an $\mathrm{Hb}$ level of $9 \mathrm{~g} / \mathrm{dL}$ or even $10 \mathrm{~g} / \mathrm{dL}$ [4].

There have also been reports related to target $\mathrm{Hb}$ levels in ESA therapy and outcomes in NDCKD patients from a series of large randomized-controlled trials (RCTs), including the CHOIR [5], CREATE [6], and TREAT [7] studies. In these studies, setting the target $\mathrm{Hb}$ level higher than $13 \mathrm{~g} / \mathrm{dL}$ in NDCKD patients gave no benefit in preserving renal function but increased the incidence of cardiovascular and stroke events. In the CREATE study, more patients progressed to dialysis when the target $\mathrm{Hb}$ level was $13.5 \mathrm{~g} / \mathrm{dL}$ than when it was $11.3 \mathrm{~g} / \mathrm{dL}$. Based on those results, the 2012 Kidney Disease: Improving Global Outcomes (KDIGO) Guidelines set an $\mathrm{Hb}$ target of 10-11.5 g/dL for NDCKD patients [9].

Nevertheless, secondary analyses of those RCTs drew attention to the impact of ESA hyporesponsiveness on outcome by showing that patients who received high ESA doses without achieving the target $\mathrm{Hb}$ level had worse outcomes [10-12]. The patients in those RCTs received higher ESA doses and experienced more cardiovascular comorbidities than NDCKD patients in Japan, so it was considered unreasonable to directly extrapolate those results to the management of anemia in Japanese NDCKD patients. In Japan, an observational study of dialysis patients [8] and a small interventional study in NDCKD patients [13] found that outcomes in patients with $\mathrm{Hb}$ levels controlled to $\geq 12 \mathrm{~g} / \mathrm{dL}$ were not inferior to those in patients with $\mathrm{Hb}$ levels between 10 and $12 \mathrm{~g} / \mathrm{dL}$. The "Guidelines for Renal Anemia in Chronic Kidney Disease" of the Japanese Society for Dialysis Therapy (JSDT) set an $\mathrm{Hb}$ target of 11-13 g/dL for NDCKD patients [14], which is higher than that in guidelines published in other countries. The above RCTs used epoetin or darbepoetin alfa, but there have been no large-scale studies of the renal protective effect of improving anemia using epoetin beta pegol (continuous erythropoietin receptor activator, C.E.R.A.), which has different erythropoietin receptor-binding properties.

Based on the above considerations, a large-scale prospective investigation of the relationship between $\mathrm{Hb}$ level achieved during C.E.R.A. treatment and the occurrence of renal events in Japanese NDCKD patients with renal anemia was conducted to investigate the effects of anemia treatment on renal outcome in the real-world clinical setting in Japan.

\section{Methods}

\section{Study design and population}

This was a prospective observational study conducted in 749 institutions in Japan. Patients were enrolled from July 2012 until December 2013, and the inclusion and exclusion criteria were as follows. Inclusion criteria: patients who are (1) NDCKD patients with renal anemia $(\mathrm{Hb}<11 \mathrm{~g} / \mathrm{dL})$, (2) receiving C.E.R.A., for the first time, regardless of history of ESA treatment, (3) not expected to transition to dialysis within 6 months from start of C.E.R.A. treatment, and (4) enrolled within 30 days after the start of C.E.R.A. treatment. Exclusion criteria: Patients who (1) have non-renal anemia, (2) had a kidney transplant in the previous year, or (3) have an estimated glomerular filtration rate (eGFR) of $<6 \mathrm{~mL} / \mathrm{min} / 1.73 \mathrm{~m}^{2}$.

\section{Anemia treatment by C.E.R.A.}

C.E.R.A. (Mircera ${ }^{\circledR} 25,50,75,100,150,200$, and $250 \mu \mathrm{g}$, Chugai Pharmaceutical, Tokyo, Japan) was used to treat renal anemia in this study in accordance with the dosage and administration method specified in the Japanese package insert. Target $\mathrm{Hb}$ levels were set at each physician's discretion.

\section{Measurements}

Data collected in this study: patient baseline characteristics (age, sex, CKD etiology, medical history, comorbidities); laboratory test data, and measurement dates; details of administration of C.E.R.A. and concomitant medication (Table 1); renal replacement therapy (RRT) date; adverse events.

\section{Endpoint}

The endpoint was renal survival, defined as time until any of the following occurs: progression to RRT, serum creatinine doubling, or eGFR falling below $6 \mathrm{~mL} / \mathrm{min} / 1.73 \mathrm{~m}^{2}$. New adverse drug reactions (ADRs) after the start of C.E.R.A. treatment were also evaluated.

The observation period was 2 years after the start of C.E.R.A. treatment but was terminated earlier if any of the following occurred: transition to RRT, death, or discontinuation of C.E.R.A. at the physician's discretion (excluding temporary suspensions).

\section{Statistical analysis}

$\mathrm{Hb}$ levels have been reported to become steady about 12 weeks after the start of C.E.R.A. treatment [15], so, in line with the protocol, landmark analysis of the effect of Week 
Table 1 Patient baseline characteristics

\begin{tabular}{|c|c|c|c|c|c|}
\hline \multirow[t]{3}{*}{ Variable } & \multicolumn{4}{|l|}{$N(\%)$} & \\
\hline & \multirow{2}{*}{$\begin{array}{l}\text { Safety analysis set } \\
(N=4601)\end{array}$} & \multirow{2}{*}{$\begin{array}{l}\text { Landmark analysis } \\
\text { set }(N=2851)\end{array}$} & \multicolumn{3}{|c|}{ Week $12 \mathrm{Hb}(\mathrm{g} / \mathrm{dL})$} \\
\hline & & & $<11(N=1793)$ & $\geq 11(N=1058)$ & $P$ value \\
\hline \multicolumn{6}{|l|}{ Sex } \\
\hline Male & $2593(56.35)$ & $1617(56.71)$ & $998(55.66)$ & $619(58.50)$ & 0.1384 \\
\hline \multicolumn{6}{|l|}{ Age (years) } \\
\hline$N$ & 4601 & 2851 & 1793 & 1058 & 0.9586 \\
\hline Mean \pm SD & $73.07 \pm 12.59$ & $72.71 \pm 12.53$ & $72.70 \pm 12.78$ & $72.72 \pm 12.11$ & \\
\hline \multicolumn{6}{|l|}{ BMI $\left(\mathrm{kg} / \mathrm{m}^{2}\right)$} \\
\hline$N$ & 2565 & 1636 & 1009 & 627 & 0.0184 \\
\hline Mean \pm SD & $23.05 \pm 4.54$ & $23.07 \pm 4.82$ & $23.29 \pm 5.40$ & $22.71 \pm 3.69$ & \\
\hline History of smoking & $1060(23.03)$ & $669(23.46)$ & $403(22.47)$ & $266(25.14)$ & 0.0726 \\
\hline \multicolumn{6}{|l|}{ CKD etiology } \\
\hline Chronic glomerulonephritis & $903(19.62)$ & $567(19.88)$ & $371(20.69)$ & $196(18.52)$ & 0.2152 \\
\hline Diabetic nephropathy & $1595(34.66)$ & $1018(35.70)$ & $649(36.19)$ & $369(34.87)$ & \\
\hline Nephrosclerosis & $1417(30.79)$ & $837(29.35)$ & $504(28.10)$ & $333(31.47)$ & \\
\hline Other & $686(14.90)$ & $429(15.04)$ & $269(15.00)$ & $160(15.12)$ & \\
\hline History of ESA therapy (last before C.E.R.A.) & $1314(28.55)$ & $844(29.60)$ & $547(30.50)$ & $297(28.07)$ & 0.1687 \\
\hline rHuEPO & $331(7.19)$ & $218(7.64)$ & $116(6.46)$ & $102(9.64)$ & \\
\hline Darbepoetin $\alpha$ & $982(21.34)$ & $625(21.92)$ & $430(23.98)$ & $195(18.43)$ & \\
\hline Unknown & $1(0.02)$ & $1(0.03)$ & $1(0.05)$ & - & \\
\hline Comorbidities & $4410(95.84)$ & $2750(96.45)$ & $1730(96.48)$ & $1020(96.40)$ & 0.9133 \\
\hline Heart disease & $1422(30.90)$ & $861(30.19)$ & $572(31.90)$ & $289(27.31)$ & \\
\hline Heart failure & $646(14.04)$ & $373(13.08)$ & $253(14.11)$ & $120(11.34)$ & \\
\hline Angina pectoris & $539(11.71)$ & $318(11.15)$ & $212(11.82)$ & $106(10.01)$ & \\
\hline Myocardial infarction & $81(1.76)$ & $51(1.78)$ & $36(2.00)$ & $15(1.41)$ & \\
\hline Arrhythmia & $369(8.01)$ & $237(8.31)$ & $161(8.97)$ & $76(7.18)$ & \\
\hline Other & $313(6.80)$ & $202(7.08)$ & $131(7.30)$ & $71(6.71)$ & \\
\hline Brain disease & $353(7.67)$ & $229(8.03)$ & $149(8.31)$ & $80(7.56)$ & \\
\hline Ischemic brain disease & $310(6.73)$ & $202(7.08)$ & $132(7.36)$ & $70(6.61)$ & \\
\hline Other & $50(1.08)$ & $32(1.12)$ & $21(1.17)$ & $11(1.03)$ & \\
\hline Hypertension & $3789(82.35)$ & $2394(83.97)$ & $1506(83.99)$ & $888(83.93)$ & \\
\hline Malignant tumor & $188(4.08)$ & $123(4.31)$ & $88(4.90)$ & $35(3.30)$ & \\
\hline Diabetes & $2051(44.57)$ & $1319(46.26)$ & $828(46.17)$ & $491(46.40)$ & \\
\hline Hyperlipidemia & $1929(41.92)$ & $1270(44.54)$ & $782(43.61)$ & $488(46.12)$ & \\
\hline Concomitant medication & $4072(88.50)$ & $2565(89.96)$ & $1604(89.45)$ & $961(90.83)$ & 0.2385 \\
\hline Iron formulation & $555(12.06)$ & $333(11.68)$ & $192(10.70)$ & $141(13.32)$ & \\
\hline Antihypertensive & $3654(79.41)$ & $2309(80.98)$ & $1453(81.03)$ & $856(80.90)$ & \\
\hline ACEI & $347(7.54)$ & $227(7.96)$ & $141(7.86)$ & $86(8.12)$ & \\
\hline ARB & $2114(45.94)$ & $1346(47.21)$ & $847(47.23)$ & $499(47.16)$ & \\
\hline Calcium channel blocker & $2528(54.94)$ & $1608(56.40)$ & $1015(56.60)$ & $593(56.04)$ & \\
\hline Diuretic & $1671(36.31)$ & $1077(37.77)$ & $691(38.53)$ & $386(36.48)$ & \\
\hline Alpha blocker & $521(11.32)$ & $337(11.82)$ & $226(12.60)$ & $111(10.49)$ & \\
\hline Beta blocker & $262(5.69)$ & $163(5.71)$ & $98(5.46)$ & $65(6.14)$ & \\
\hline Other & $1053(22.88)$ & $687(24.09)$ & $440(24.53)$ & $247(23.34)$ & \\
\hline Active vitamin $\mathrm{D}_{3}$ analog & $403(8.75)$ & $244(8.55)$ & $136(7.58)$ & $108(10.20)$ & \\
\hline Hypolipidemic drug & $1476(32.07)$ & $982(34.44)$ & $611(34.07)$ & $371(35.06)$ & \\
\hline Antidiabetic drug & $1250(27.16)$ & $828(29.04)$ & $512(28.55)$ & $316(29.86)$ & \\
\hline \multicolumn{6}{|l|}{ Systolic blood pressure (mmHg) } \\
\hline$N$ & 4055 & 2541 & 1599 & 942 & 0.0041 \\
\hline
\end{tabular}


Table 1 (continued)

\begin{tabular}{|c|c|c|c|c|c|}
\hline \multirow[t]{3}{*}{ Variable } & \multicolumn{4}{|l|}{$N(\%)$} & \\
\hline & \multirow{2}{*}{$\begin{array}{l}\text { Safety analysis set } \\
(N=4601)\end{array}$} & \multirow{2}{*}{$\begin{array}{l}\text { Landmark analysis } \\
\text { set }(N=2851)\end{array}$} & \multicolumn{3}{|c|}{ Week $12 \mathrm{Hb}(\mathrm{g} / \mathrm{dL})$} \\
\hline & & & $<11(N=1793)$ & $\geq 11(N=1058)$ & $P$ value \\
\hline Mean \pm SD & $134.94 \pm 20.45$ & $134.60 \pm 20.14$ & $135.48 \pm 20.62$ & $133.11 \pm 19.21$ & \\
\hline \multicolumn{6}{|c|}{ Diastolic blood pressure $(\mathrm{mmHg})$} \\
\hline$N$ & 4028 & 2524 & 1589 & 935 & 0.8740 \\
\hline Mean \pm SD & $70.09 \pm 12.82$ & $70.16 \pm 12.73$ & $70.12 \pm 12.97$ & $70.21 \pm 12.31$ & \\
\hline \multicolumn{6}{|l|}{$\mathrm{Hb}(\mathrm{g} / \mathrm{dL})$} \\
\hline$N$ & 4601 & 2851 & 1793 & 1058 & $<0.0001$ \\
\hline Mean \pm SD & $9.45 \pm 0.92$ & $9.48 \pm 0.90$ & $9.27 \pm 0.90$ & $9.83 \pm 0.77$ & \\
\hline \multicolumn{6}{|c|}{ Serum creatinine $(\mathrm{mg} / \mathrm{dL})$} \\
\hline$N$ & 4601 & 2851 & 1793 & 1058 & 0.0001 \\
\hline Mean \pm SD & $2.87 \pm 1.38$ & $2.85 \pm 1.30$ & $2.92 \pm 1.32$ & $2.73 \pm 1.25$ & \\
\hline \multicolumn{6}{|c|}{ eGFR (mL/min/1.73 m²) } \\
\hline$N$ & 4601 & 2851 & 1793 & 1058 & 0.0004 \\
\hline Mean \pm SD & $20.47 \pm 11.67$ & $20.25 \pm 11.08$ & $19.69 \pm 10.94$ & $21.21 \pm 11.25$ & \\
\hline \multicolumn{6}{|c|}{ Total protein $(\mathrm{g} / \mathrm{dL})$} \\
\hline$N$ & 3763 & 2346 & 1472 & 874 & 0.3386 \\
\hline Mean \pm SD & $6.64 \pm 0.72$ & $6.65 \pm 0.74$ & $6.64 \pm 0.74$ & $6.67 \pm 0.74$ & \\
\hline \multicolumn{6}{|l|}{ Albumin (g/dL) } \\
\hline$N$ & 3989 & 2499 & 1563 & 936 & $<0.0001$ \\
\hline Mean \pm 2 SD & $3.62 \pm 0.55$ & $3.64 \pm 0.54$ & $3.60 \pm 0.55$ & $3.70 \pm 0.53$ & \\
\hline \multicolumn{6}{|c|}{ Ferritin (ng/mL) } \\
\hline$N$ & 1853 & 1178 & 732 & 446 & 0.0089 \\
\hline Mean \pm SD & $166.94 \pm 213.14$ & $172.54 \pm 240.75$ & $158.24 \pm 206.56$ & $196.00 \pm 286.92$ & \\
\hline \multicolumn{6}{|l|}{ TSAT $(\%)$} \\
\hline$N$ & 1667 & 1062 & 661 & 401 & 0.5173 \\
\hline Mean \pm SD & $28.24 \pm 12.96$ & $28.45 \pm 12.89$ & $28.25 \pm 13.07$ & $28.78 \pm 12.60$ & \\
\hline \multicolumn{6}{|c|}{ Calcium (mg/dL) } \\
\hline$N$ & 3562 & 2263 & 1443 & 820 & 0.0002 \\
\hline Mean \pm SD & $8.80 \pm 1.22$ & $8.83 \pm 1.24$ & $8.76 \pm 1.17$ & $8.96 \pm 1.34$ & \\
\hline \multicolumn{6}{|c|}{ Phosphate (mg/dL) } \\
\hline$N$ & 3158 & 2016 & 1276 & 740 & $<0.0001$ \\
\hline Mean \pm SD & $3.94 \pm 1.05$ & $3.93 \pm 1.13$ & $4.01 \pm 1.30$ & $3.80 \pm 0.74$ & \\
\hline \multicolumn{6}{|c|}{ Total cholesterol (mg/dL) } \\
\hline$N$ & 2770 & 1728 & 1093 & 635 & 0.0146 \\
\hline Mean \pm SD & $171.88 \pm 42.05$ & $171.93 \pm 42.02$ & $170.04 \pm 39.84$ & $175.16 \pm 45.39$ & \\
\hline \multicolumn{6}{|l|}{ CRP (mg/dL) } \\
\hline$N$ & 2456 & 1526 & 966 & 560 & 0.9413 \\
\hline Mean \pm SD & $0.58 \pm 1.57$ & $0.52 \pm 1.39$ & $0.52 \pm 1.37$ & $0.53 \pm 1.43$ & \\
\hline \multicolumn{6}{|c|}{ Urine protein (spot urine) (mg/dL) } \\
\hline$N$ & 2321 & 1494 & 919 & 575 & 0.1941 \\
\hline Mean \pm SD & $183.34 \pm 256.55$ & $187.57 \pm 243.26$ & $194.04 \pm 238.07$ & $177.24 \pm 251.20$ & \\
\hline \multicolumn{6}{|c|}{ Urine protein/creatinine ratio } \\
\hline$N$ & 2020 & 1297 & 794 & 503 & 0.0274 \\
\hline Mean \pm SD & $5.19 \pm 34.08$ & $5.28 \pm 33.78$ & $6.93 \pm 42.93$ & $2.68 \pm 4.91$ & \\
\hline \multicolumn{6}{|c|}{ C.E.R.A. dosage ( $\mu \mathrm{g} / 4$ week) } \\
\hline$N$ & 4594 & 2848 & 1792 & 1056 & 0.1528 \\
\hline Mean \pm SD & $66.82 \pm 48.99$ & $66.35 \pm 47.73$ & $65.37 \pm 44.74$ & $68.01 \pm 52.39$ & \\
\hline
\end{tabular}


Table 1 (continued)

N, number of patiens; SD, standard deviation; BMI, body mass index; CKD, chronic kidney disease; ESA, erythropoiesis-stimulating agent; C.E.R.A., continuous erythropoietin receptor activator; rHuEPO; recombinant human erythropoietin; ACEI, angiotensin-converting enzyme inhibitor; ARB, angiotensin receptor blocker; Hb, Hemoglobin; eGFR, estimated glomerular filtration rate; TSAT, transferrin saturation; CRP,Creactive protein

$12 \mathrm{Hb}$ level on the occurrence of renal events was performed with week 12 as the landmark time (start time).

The landmark analysis set was the safety analysis set minus patients who experienced renal events were censored in treatment Weeks 0 to 12, or had missing data on $\mathrm{Hb}$ level in treatment of week 12 (Week $12 \mathrm{Hb}$ ) (Fig. 1). The landmark analysis set was divided into two groups based on Week $12 \mathrm{Hb}(<11 \mathrm{~g} / \mathrm{dL}, \geq 11 \mathrm{~g} / \mathrm{dL})$, and renal survival rates were estimated by the Kaplan-Meier analysis and compared by log-rank test. In addition, univariate and multivariate Cox regression analyses were used to estimate the risk of week $12 \mathrm{Hb}$ levels for renal outcomes using hazard ratio (HR) and 95\% confidence interval [CI] (Table 2 ).

All analyses were performed using SAS version 9.4 (SAS Institute, Cary, NC, USA), and values of $P<0.05$ were considered significant.

\section{Results}

\section{Patients and baseline characteristics}

Of 5305 patients enrolled, 254 for whom case report forms were not retrieved and 450 who did not meet inclusion criteria or met exclusion criteria were excluded, leaving 4601 in the safety analysis set. After excluding 1750 patients who had renal events or were censored by week 12 or who had missing data on week $12 \mathrm{Hb}$, the landmark analysis set of 2851 patients was established (Fig. 1). In these patients, the median observation period was 66.57 weeks. The landmark analysis set was divided into two groups based on week 12 $\mathrm{Hb}$ : the $<11 \mathrm{~g} / \mathrm{dL}$ group had 1793 patients and the $\geq 11 \mathrm{~g} / \mathrm{dL}$ group had 1058 patients. The median observation period was 80.14 weeks in the group with $\mathrm{Hb}$ level $\geq 11 \mathrm{~g} / \mathrm{dL}$ and 60.00 weeks in the group with $\mathrm{Hb}$ level $<11 \mathrm{~g} / \mathrm{dL}$. The final observation of cumulative renal survival rate was at 158.14 weeks in the group with $\mathrm{Hb}$ level $\geq 11 \mathrm{~g} / \mathrm{dL}$ and 164.14 weeks in the group with $\mathrm{Hb}$ level $<11 \mathrm{~g} / \mathrm{dL}$.

The patient baseline characteristics are shown in Table 1. Of the whole landmark analysis set, $56.7 \%$ were male, the mean age was $72.7 \pm 12.5$ years (there were many elderly patients, reflecting the clinical reality), $35.7 \%$ of the patients had diabetic nephropathy as the CKD etiology, and 29.6\% had a history of ESA therapy before receiving C.E.R.A. There were no significant differences in sex, age, CKD etiology, medical history, comorbidity, or antihypertensive agents between the two groups.
The mean week 0 and week $12 \mathrm{Hb}$ levels were respectively $9.27 \pm 0.90$ and $9.77 \pm 0.90 \mathrm{~g} / \mathrm{dL}$ in the $<11 \mathrm{~g} / \mathrm{dL}$ group and $9.83 \pm 0.77$ and $11.79 \pm 0.70 \mathrm{~g} / \mathrm{dL}$ in the $\geq 11 \mathrm{~g} /$ $\mathrm{dL}$ group. The mean week 0 and week 12 eGFR values were respectively $19.69 \pm 10.94$ and $18.98 \pm 12.00 \mathrm{~mL} /$ $\mathrm{min} / 1.73 \mathrm{~m}^{2}$ in the $<11 \mathrm{~g} / \mathrm{dL}$ group and $21.21 \pm 11.25$ and $21.02 \pm 12.01 \mathrm{~mL} / \mathrm{min} / 1.73 \mathrm{~m}^{2}$ in the $\geq 11 \mathrm{~g} / \mathrm{dL}$ group.

\section{Hb level achieved and C.E.R.A. dosage}

In the $\geq 11 \mathrm{~g} / \mathrm{dL}$ group, Hb level remained around $11 \mathrm{~g} / \mathrm{dL}$ at all time points after week 12 . In the $<11 \mathrm{~g} / \mathrm{dL}$ group, $\mathrm{Hb}$ level stayed around $10 \mathrm{~g} / \mathrm{dL}$ after week 16 .

The week 12 C.E.R.A dosage in the $\geq 11 \mathrm{~g} / \mathrm{dL}$ group $(67.80 \pm 42.47 \mu \mathrm{g} / 4$ week) was significantly lower than in the $<11 \mathrm{~g} / \mathrm{dL}$ group $(78.55 \pm 50.43 \mu \mathrm{g} / 4$ week $)(P<0.0001)$. The range of mean dosages during the observation period was $64.88-75.50 \mu \mathrm{g} / 4$ week in the $\geq 11 \mathrm{~g} / \mathrm{dL}$ group and $78.55-88.89 \mu \mathrm{g} / 4$ week in the $<11 \mathrm{~g} / \mathrm{dL}$ group, remaining roughly the same as in week 12 (Fig. 2).

\section{Endpoint}

According to landmark analysis starting from treatment week 12 , renal events occurred in 693 patients $(38.65 \%)$ in the $<11 \mathrm{~g} / \mathrm{dL}$ group and 338 patients $(31.94 \%)$ in the $\geq 11 \mathrm{~g} /$ dL group.

The overall 2 year renal survival rate by Kaplan-Meier analysis was $37.57 \%$ in the $<11 \mathrm{~g} / \mathrm{dL}$ group and $51.47 \%$ in the $\geq 11 \mathrm{~g} / \mathrm{dL}$ group, so it was significantly higher in the $\geq 11 \mathrm{~g} / \mathrm{dL}$ group $(P<0.0001$, log-rank test) (Fig. 3$)$.

Investigation of the HR for renal events using multivariate Cox regression analysis also revealed a significantly higher risk of renal events in the $<11 \mathrm{~g} / \mathrm{dL}$ group compared to the $\geq 11 \mathrm{~g} / \mathrm{dL}$ group (HR 1.26 ; 95\% CI $1.05-1.51 ; P=0.0103$ ). High eGFR, high albumin, and advanced age significantly decreased the risk of renal events, whereas high systolic blood pressure, male sex, diabetic nephropathy, calcium channel blockers, activated carbon product, sodium bicarbonate, and higher C.E.R.A. dose in week 12 significantly increased the HR for renal events (Table 2).

Kaplan-Meier analysis of the patients divided into five groups based on Week $12 \mathrm{Hb}(<9,9$ to $<10,10$ to $<11,11$ to $<12$, and $\geq 12 \mathrm{~g} / \mathrm{dL}$ ) showed that renal outcomes were poorer with $\mathrm{Hb}$ levels $<10 \mathrm{~g} / \mathrm{dL}$ (Fig. 4), and multivariate Cox regression analysis (step-down procedure) revealed a significantly higher risk of renal events in the $<9 \mathrm{~g} / \mathrm{dL}$ 
Table 2 Univariate and multivariate cox regression analyses with patients divided into two groups by treatment week $12 \mathrm{Hb}$ level

\begin{tabular}{|c|c|c|c|c|c|c|c|}
\hline \multirow[t]{2}{*}{ Variable } & & \multicolumn{3}{|c|}{ Univariate analysis } & \multicolumn{3}{|c|}{ Step-down procedure } \\
\hline & & Hazard ratio & $95 \% \mathrm{CI}$ & $P$ value & Hazard ratio & $95 \% \mathrm{CI}$ & $P$ value \\
\hline \multirow[t]{2}{*}{ Week $12 \mathrm{Hb}(\mathrm{g} / \mathrm{dL})$} & $\geq 11$ & \multicolumn{3}{|l|}{ Reference } & \multicolumn{3}{|l|}{ Reference } \\
\hline & $<11$ & 1.37 & $1.21-1.57$ & $<0.0001$ & 1.26 & $1.05-1.51$ & 0.0103 \\
\hline eGFR & $5 \mathrm{~mL} / \mathrm{min} / 1.73 \mathrm{~m}^{2}$ & 0.57 & $0.54-0.60$ & $<0.0001$ & 0.59 & $0.55-0.64$ & $<0.0001$ \\
\hline Systolic blood pressure & $10 \mathrm{mmHg}$ & 1.17 & $1.14-1.21$ & $<0.0001$ & 1.11 & $1.06-1.15$ & $<0.0001$ \\
\hline Albumin & $0.5 \mathrm{~g} / \mathrm{dL}$ & 0.71 & $0.68-0.75$ & $<0.0001$ & 0.7 & $0.65-0.75$ & $<0.0001$ \\
\hline Age & 10 years & 0.74 & $0.71-0.77$ & $<0.0001$ & 0.77 & $0.72-0.83$ & $<0.0001$ \\
\hline Sex & Male & 1.5 & $1.32-1.70$ & $<0.0001$ & 1.42 & $1.19-1.69$ & $<0.0001$ \\
\hline History of smoking & Yes & 1.41 & $1.21-1.64$ & $<0.0001$ & - & - & - \\
\hline History of ESA therapy & Yes & 1.04 & $0.91-1.19$ & 0.5148 & - & - & - \\
\hline Diet therapy & Yes & 1.65 & $1.41-1.93$ & $<0.0001$ & 1.18 & $0.94-1.48$ & 0.1442 \\
\hline \multicolumn{8}{|l|}{ Etioligy } \\
\hline Diabetic nephropathy & Yes & 1.6 & $1.41-1.81$ & $<0.0001$ & 1.25 & $1.00-1.55$ & 0.0428 \\
\hline Nephrosclerosis & Yes & 0.6 & $0.52-0.70$ & $<0.0001$ & - & - & - \\
\hline Chronic glomerulonephritis & Yes & 1.12 & $0.97-1.30$ & 0.1162 & - & - & - \\
\hline Heart disease (medical history) & Yes & 0.87 & $0.73-1.05$ & 0.1707 & - & - & - \\
\hline \multicolumn{8}{|l|}{ Comorbidity } \\
\hline Hypertension & Yes & 1.63 & $1.33-2.00$ & $<0.0001$ & - & - & - \\
\hline Heart failure & Yes & 0.89 & $0.73-1.08$ & 0.2561 & - & - & - \\
\hline Angina pectoris or myocardial infarction & Yes & 0.93 & $0.77-1.13$ & 0.4964 & - & - & - \\
\hline Diabetes & Yes & 1.39 & $1.23-1.57$ & $<0.0001$ & - & - & - \\
\hline Hyperlipidemia & Yes & 1.07 & $0.95-1.21$ & 0.2270 & - & - & - \\
\hline \multicolumn{8}{|l|}{ Concomitant medication } \\
\hline Iron formulation & Yes & 0.86 & $0.71-1.05$ & 0.1484 & 0.77 & $0.60-1.00$ & 0.0538 \\
\hline ACEIs or ARBs & Yes & 1.29 & $1.13-1.45$ & $<0.0001$ & 0.87 & $0.72-1.04$ & 0.1344 \\
\hline Calcium channel blocker & Yes & 1.9 & $1.66-2.17$ & $<0.0001$ & 1.3 & $1.07-1.59$ & 0.0069 \\
\hline Diuretic & Yes & 1.24 & $1.10-1.41$ & 0.0004 & - & - & - \\
\hline Activated carbon product & Yes & 1.67 & $1.44-1.92$ & $<0.0001$ & 1.27 & $1.04-1.54$ & 0.0156 \\
\hline Active vitamin D3 analog & Yes & 1.28 & $1.05-1.57$ & 0.0141 & 1.22 & $0.95-1.58$ & 0.1160 \\
\hline Antidiabetic drug & Yes & 1.25 & $1.09-1.42$ & 0.0007 & 0.84 & $0.67-1.04$ & 0.1216 \\
\hline Hypolipidemic drug & Yes & 1.13 & $0.99-1.28$ & 0.0558 & - & - & - \\
\hline Sodium bicarbonate & Yes & 2 & $1.69-2.37$ & $<0.0001$ & 1.46 & $1.17-1.82$ & 0.0007 \\
\hline Treatment Week 12 C.E.R.A. dosage & $25 \mu \mathrm{g} / 4$ week & 1.08 & $1.05-1.11$ & $<0.0001$ & 1.06 & $1.02-1.10$ & 0.0025 \\
\hline
\end{tabular}

Among variables for which $<20 \%$ of the data were missing, the above clinically significant risk factors that could conceivably be confounding factors were investigated as covariates. Multivariate Cox regression analysis was performed using a model in which covariates were selected by the step-down procedure (excluding covariates for which $P$ was $\geq 0.2$ )

CI, confidence interval; Hb, Hemoglobin; eGFR, estimated glomerular filtration rate; ESA, erythropoiesis-stimulating agent; ACEI, angiotensinconverting enzyme inhibitor; ARB, angiotensin receptor blocker; C.E.R.A., continuous erythropoietin receptor activator

group (HR 1.89; 95\% CI 1.39-2.56; $P<0.0001)$ and the $9-<10 \mathrm{~g} / \mathrm{dL}$ group (HR 1.52; 95\% CI 1.19-1.94; $P=0.0006$ ) compared to the $11-<12 \mathrm{~g} / \mathrm{dL}$ (reference) group (data not shown).

To investigate the effect of the Hb level not only at a single point (Week 12) but also during its subsequent course, the relationship between length of time with $\mathrm{Hb} \geq 11 \mathrm{~g} / \mathrm{dL}$ after start of C.E.R.A. treatment and renal events was investigated using multivariate Cox regression analysis. Dividing the patients into three groups based on time with $\mathrm{Hb} \geq 11 \mathrm{~g} / \mathrm{dL}$ during the first year of C.E.R.A. treatment ( $<3$ month, 3 to $<6$ month, and 6-12 month) and analyzing the risk of renal events occurring after the first year of treatment revealed a significantly lower risk of renal events in the 6-12 month group (HR 0.72; 95\% CI $0.55-0.93 ; P=0.0135)$ compared to the $<3$ month group (data not shown). 


\begin{tabular}{|c|c|c|c|c|}
\hline Enrolled & 5305 patients & & & \\
\hline & & \multicolumn{2}{|c|}{ Case report form not recoverable } & 254 patients \\
\hline Case report form recovered & 5051 patients & & & \\
\hline & & \multirow{5}{*}{$\begin{array}{c}\text { Excluded from } \\
\text { safety analysis set } \\
450 \text { patients } \\
\text { (some overlap) }\end{array}$} & Previously received C.E.R.A. & 7 patients \\
\hline & & & Outside enrollment period & 29 patients \\
\hline & & & Baseline $\mathrm{Hb} \geq 11 \mathrm{~g} / \mathrm{dL}$ or missing & 282 patients \\
\hline & & & Baseline eGFR $<6 \mathrm{~mL} / \mathrm{min} / 1.73 \mathrm{~m}^{2}$ or missing & 214 patients \\
\hline & & & Nonrenal anemia & 60 patients \\
\hline
\end{tabular}

\begin{tabular}{|c|c|c|c|c|}
\hline Safety analysis set & 4601 patients & & & \\
\hline & & \multirow{3}{*}{$\begin{array}{l}\text { Excluded from } \\
\text { analysis set } \\
1750 \text { patients } \\
\text { (some overlap) }\end{array}$} & Renal events by Week 12 & 216 patients \\
\hline & & & Censored by Week 12 & 447 patients \\
\hline & & & Week $12 \mathrm{Hb}$ missing & 1568 patients \\
\hline
\end{tabular}

\section{\begin{tabular}{l|l|} 
Landmark analysis set & 2851 patients \\
\hline
\end{tabular}}

Fig. 1 Subject disposition diagram. C.E.R.A., continuous erythropoietin receptor activator; Hb, Hemoglobin; eGFR, estimated glomerular filtration rate

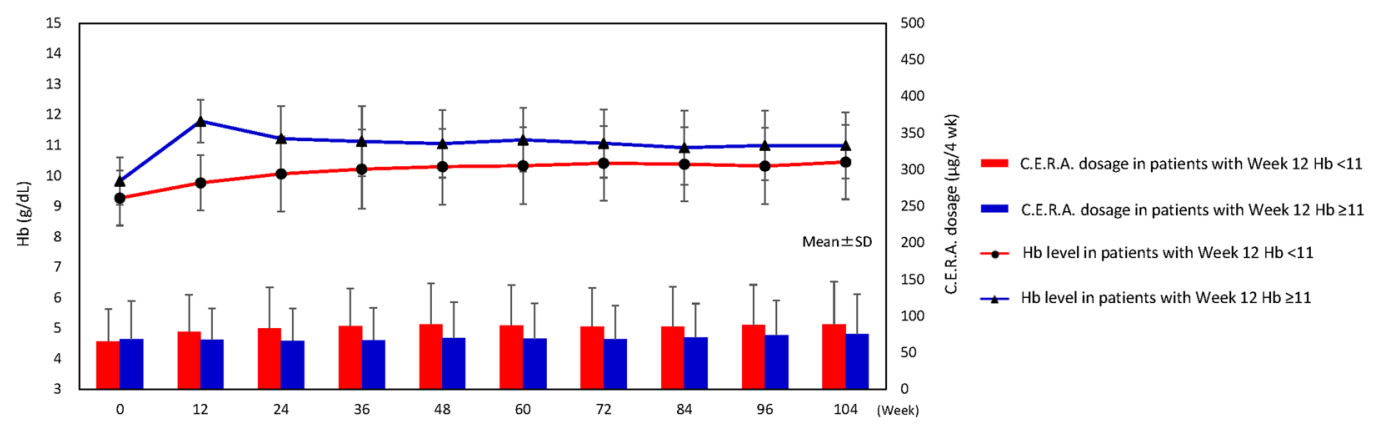

\begin{tabular}{|c|c|c|c|c|c|c|c|c|c|c|c|}
\hline \multirow[t]{2}{*}{$\mathrm{N}$} & & \multicolumn{10}{|c|}{ Observation time (week) } \\
\hline & & 0 & 12 & 24 & 36 & 48 & 60 & 72 & 84 & 96 & 104 \\
\hline \multirow[t]{2}{*}{$\mathrm{Hb}(\mathrm{g} / \mathrm{dL})$} & Week $12 \mathrm{Hb}<11$ & 1793 & 1793 & 1236 & 941 & 844 & 700 & 619 & 559 & 504 & 455 \\
\hline & Week $12 \mathrm{Hb} \geq 11$ & 1058 & 1058 & 761 & 618 & 556 & 477 & 439 & 399 & 359 & 324 \\
\hline \multirow{2}{*}{ C.E.R.A. dosage $(\mu \mathrm{g} / 4 \mathrm{wk})$} & Week $12 \mathrm{Hb}<11$ & 1792 & 1707 & 1414 & 1131 & 1016 & 860 & 772 & 690 & 627 & 498 \\
\hline & Week $12 \mathrm{Hb} \geq 11$ & 1056 & 951 & 828 & 715 & 651 & 579 & 527 & 485 & 444 & 347 \\
\hline
\end{tabular}

Fig. 2 Time courses of C.E.R.A. dosage and Hb level with patients divided into two groups by treatment week 12 Hb level. C.E.R.A., continuous erythropoietin receptor activator; Hb, Hemoglobin; $\mathrm{N}$, number of patients; SD, standard deviation

\section{Subgroup analyses}

The effect of Week $12 \mathrm{Hb}$ on the occurrence of renal events was also investigated in CKD etiology and age subgroups. Kaplan-Meier analysis showed that patients in the $\geq 11 \mathrm{~g} / \mathrm{dL}$ group had significantly lower risk of renal events compared to the $<11 \mathrm{~g} / \mathrm{dL}$ group across the subgroups with or without diabetic nephropathy, with or without nephrosclerosis, and with age $\geq 75$ or $<75$ years (see Fig. 5 for $P$ values).

Multivariate Cox regression analyses also showed that favorable effects of higher Week $12 \mathrm{Hb}$ level on renal outcomes were observed across patient subgroups with age $\geq 75$ or $<75$ years $(P=0.635$ for interaction) and with or without nephrosclerosis ( $P=0.261$ for interaction). However, a similar effect was not observed in the patient subgroups with 


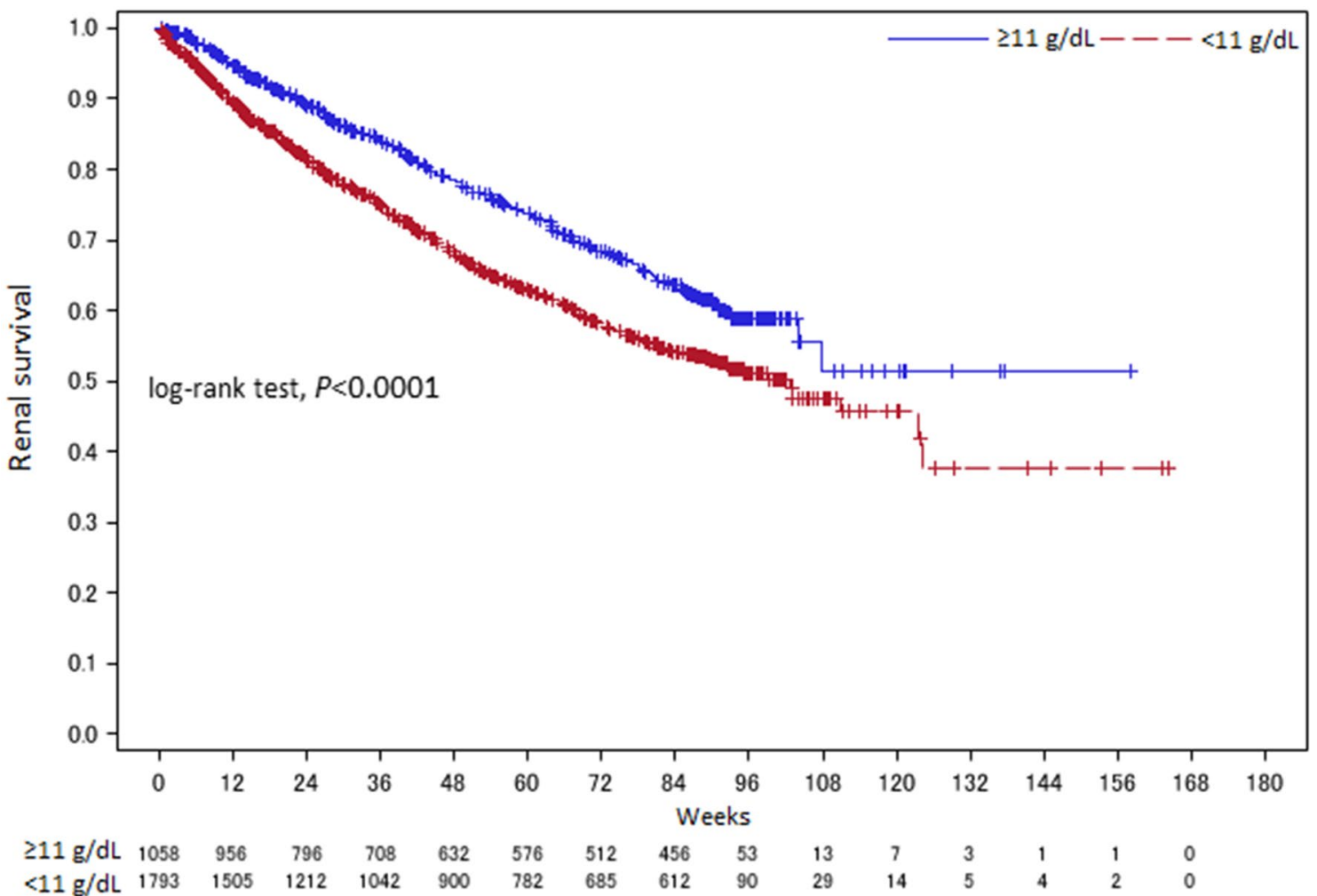

Fig. 3 Kaplan-Meier analysis with patients divided into two groups by treatment Week $12 \mathrm{Hb}$ level. Week 0: C.E.R.A. treatment week 12 was used as the landmark time (start time)

or without diabetic nephropathy ( $P=0.003$ for interaction) (Fig. 6).

\section{Safety analyses}

ADRs occurred in 195 (4.23\%) of the 4601 patients in the safety analysis set. Among the 2851 patients in the landmark analysis set, ADRs occurred in 77 patients $(4.29 \%)$ in the $<11 \mathrm{~g} / \mathrm{dL}$ group and 45 patients $(4.25 \%)$ in the $\geq 11 \mathrm{~g} / \mathrm{dL}$ group; Hb level did not affect the incidence of ADRs. Serious ADRs occurred in 39 patients $(2.17 \%)$ in the $<11 \mathrm{~g} /$ $\mathrm{dL}$ group and 22 patients $(2.07 \%)$ in the $\geq 11 \mathrm{~g} / \mathrm{dL}$ group (Table 3).

\section{Discussion}

The relationship between treating renal anemia with ESAs and renal outcomes in NDCKD patients has been investigated in various observational studies and secondary analyses of large-scale RCTs over the past 2 decades. The evidence level is high, particularly from large-scale RCTs (e.g., the CHOIR, CREATE, and TREAT), but this evidence cannot be directly extrapolated to the Japanese clinical setting, because the high ESA doses and very high rates of cardiovascular comorbidities in these studies differ greatly from those found in Japan. Furthermore, because these studies used epoetin and darbepoetin alfa, the effect of C.E.R.A. to treat renal anemia on renal outcome remained unclear. $\mathrm{We}$, therefore, conducted a large-scale prospective observational study to clarify this effect in Japanese NDCKD patients under actual-use conditions.

The results confirmed two crucial points. The first is that landmark analysis of week $12 \mathrm{Hb}$ showed a significantly lower risk of renal events in the $\geq 11 \mathrm{~g} / \mathrm{dL}$ group compared to the $<11 \mathrm{~g} / \mathrm{dL}$ group (Fig. 3; Table 2). Furthermore, dividing the patients into five groups based on week $12 \mathrm{Hb}$ revealed significantly higher risk of renal events in the $<9$ and 9 to $<10 \mathrm{~g} / \mathrm{dL}$ groups compared to the $11-<12 \mathrm{~g} / \mathrm{dL}$ reference group (Fig. 4). We, therefore, consider favorable renal outcomes more likely if the $\mathrm{Hb}$ level can be kept to at least $\geq 10 \mathrm{~g} / \mathrm{dL}$ and ideally $\geq 11 \mathrm{~g} / \mathrm{dL}$. Investigation of the relationship between the length of time that $\mathrm{Hb}$ was $\geq 11 \mathrm{~g} /$ $\mathrm{dL}$ and the onset of renal events suggested that achieving an $\mathrm{Hb}$ level $\geq 11 \mathrm{~g} / \mathrm{dL}$ for $\geq 6$ months with C.E.R.A. further decreases the risk of renal events, as shown by Luca et al. [16]. Although the $2012 \mathrm{KDIGO}$ guidelines [9] decreased the upper limit of the target $\mathrm{Hb}$ level to $11.5 \mathrm{~g} / \mathrm{dL}$, emphasizing the results of the CHOIR, CREATE, and TREAT studies, our results support the target maintenance $\mathrm{Hb}$ level in 


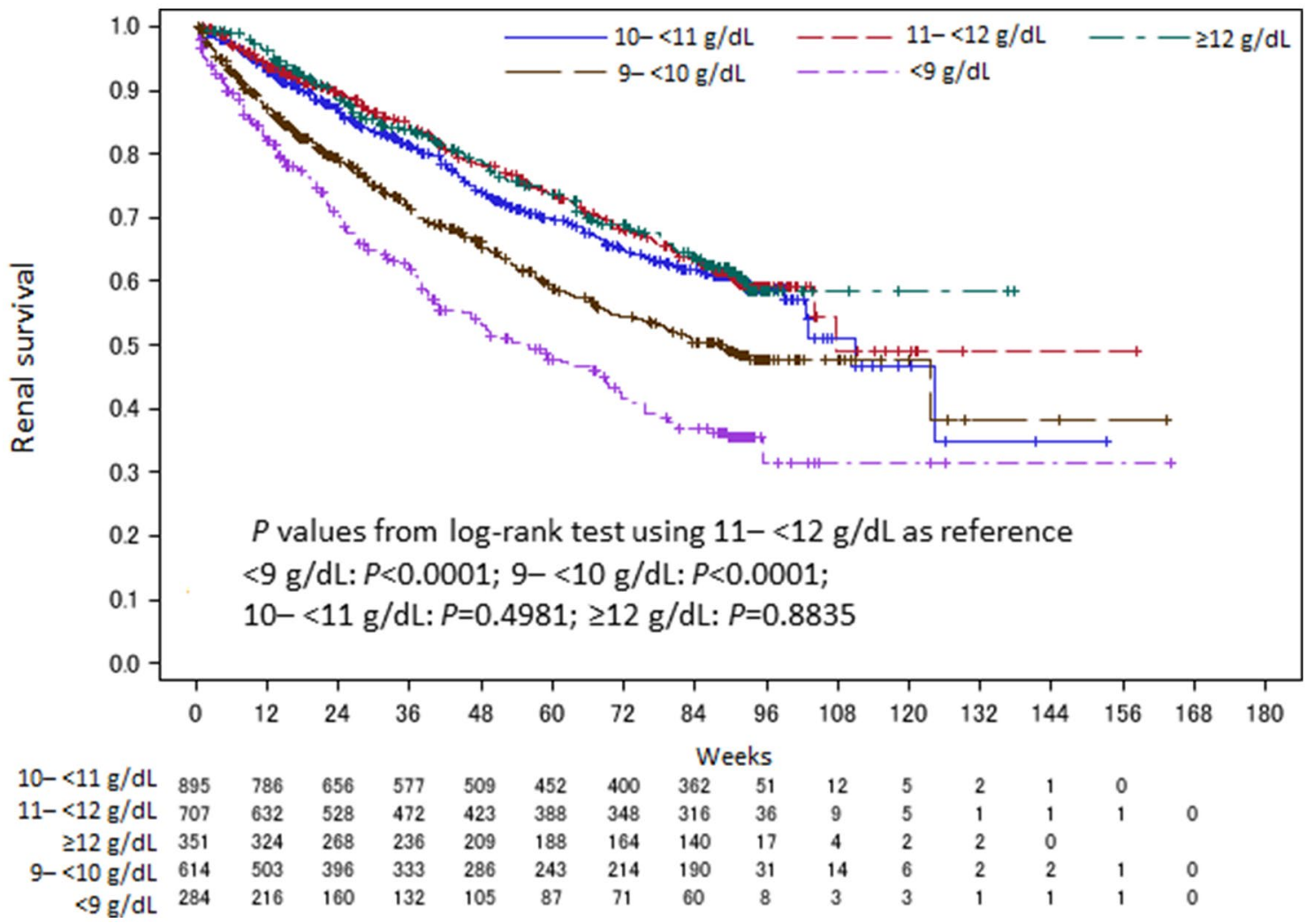

Fig. 4 Kaplan-Meier analysis with patients divided into five groups by treatment Week $12 \mathrm{Hb}$ level. The overall renal survival rate by Kaplan-Meier analysis was $31.43 \%$ in the $<9 \mathrm{~g} / \mathrm{dL}$ group, $38.08 \%$ in the 9 to $<10 \mathrm{~g} / \mathrm{dL}$ group, $34.98 \%$ in the 10 to $<11 \mathrm{~g} / \mathrm{dL}$ group,

NDCKD patients ( 11 to $<13 \mathrm{~g} / \mathrm{dL}$ ) stated in the 2015 JSDT "Guidelines for Renal Anemia in Chronic Kidney Disease" [14] for patient populations (such as CKD patients in Japan) that receive lower ESA doses and have fewer cardiovascular comorbidities than CKD patients in the United States.

Furthermore, in multivariate Cox regression analysis of subgroups with or without diabetic nephropathy, with or without nephrosclerosis, and with age $\geq 75$ or $<75$ years, interaction was observed in the patient subgroups with or without diabetic nephropathy ( $P=0.003$ for interaction) (Fig. 6). The status of atherosclerotic lesions in multiple organs including the kidneys may differ in the patient subgroups with or without diabetic nephropathy; it might have affected this interaction. In addition, it seems to be relevant that higher $\mathrm{Hb}$ level would cancel the benefit from ESA treatment as previously shown in the CHOIR and TREAT studies. This could warrant further investigation of the usefulness of achieving an $\mathrm{Hb}$ level $\geq 11 \mathrm{~g} / \mathrm{dL}$ to delay renal outcomes in these patient groups.

The second point is that at Week 12 C.E.R.A dosage in the $\geq 11 \mathrm{~g} / \mathrm{dL}$ group $(67.80 \pm 42.47 \mu \mathrm{g} / 4$ week $)$ was significantly lower than that in the $<11 \mathrm{~g} / \mathrm{dL}$ group $(78.55 \pm 50.43 \mu \mathrm{g} / 4$ week) $(P<0.0001)$. And, thereafter, they remained roughly
$49.00 \%$ in the 11 to $<12 \mathrm{~g} / \mathrm{dL}$ group, and $58.60 \%$ in the $\geq 12 \mathrm{~g} / \mathrm{dL}$ group. Week 0: C.E.R.A. treatment Week 12 was used as the landmark time (start time). Hb, Hemoglobin; $\mathrm{N}$, number of patients

the same as in week 12 (Fig. 2). Recently, Tsuruya et al. reported the results of a pooled analysis of C.E.R.A. clinical studies in Japan. They divided patients into two groups based on median erythropoietin resistance index (ERI) in Week 12 of C.E.R.A. treatment, as a measure of ESA responsiveness, and found that renal outcome (initiation of dialysis or $30 \%$ decrease in eGFR) was significantly poorer in the poorresponse group compared to the good-response group [17]. Our study did not investigate ERI, but the C.E.R.A. dose in the $\geq 11 \mathrm{~g} / \mathrm{dL}$ group tended to be lower than in the $<11 \mathrm{~g} /$ $\mathrm{dL}$ group throughout the observation period. This suggests a possible relationship between C.E.R.A. responsiveness and renal outcome. We are aware that ESA hyporesponsiveness is an unstudied issue of ESA therapy for renal anemia and look forward to the results of a prospective comparative study of the relationship between ERI and improvement of renal outcome.

In terms of safety evaluation, there were no major differences in the incidence of ADRs related to elevated $\mathrm{Hb}$ (e.g., myocardial infarction, angina pectoris, cerebral infarction, cerebral hemorrhage, aortic dissection, and hypertension). Therefore, achieving an $\mathrm{Hb}$ level $\geq 11 \mathrm{~g} / \mathrm{dL}$ is not considered to present any safety issues in actual clinical practice. 

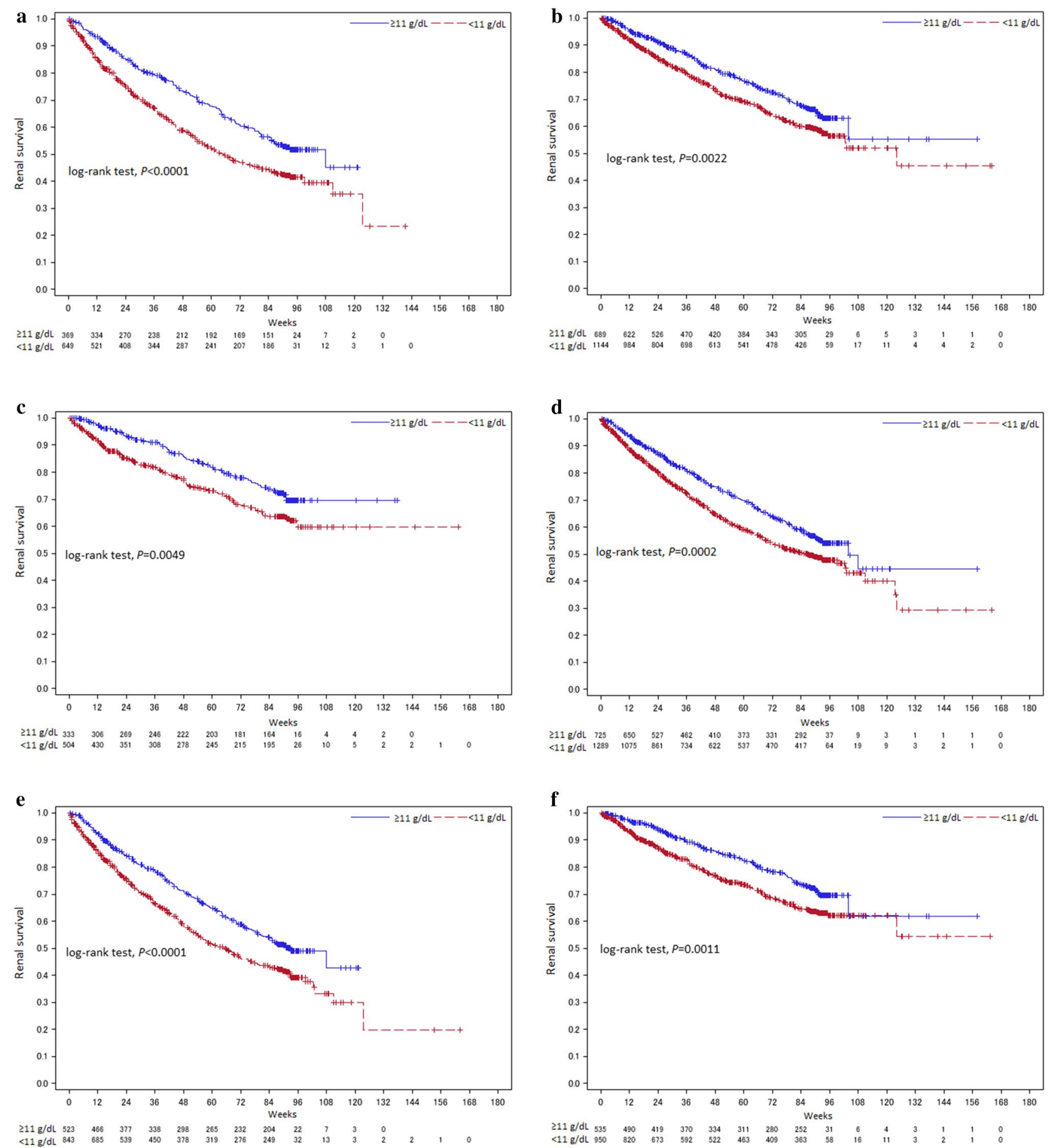

These results should be interpreted with the study's limitations in mind. First, this was an observational study, so setting the target $\mathrm{Hb}$ level was the responsibility of the physicians, not the result of an intervention. Furthermore, we cannot exclude the possibility of reverse causality regarding several covariates (calcium channel blockers, activated carbon product, and sodium bicarbonate) used in the multivariate Cox regression analysis. Second,

although the patient baseline characteristics, Hb levels, and other data, shown in Table 2, were adjusted for using multivariate Cox regression analysis, adjustment for urine protein, urine creatinine, and C-reactive protein was not performed, because $\geq 20 \%$ of the data were missing. If factors with $\geq 20 \%$ of data missing are included in analysis as covariates, it can introduce bias in multivariate Cox regression analysis results obtained for the remaining 
4Fig. 5 Kaplan-Meier analysis (by CKD etiology and age) with patients divided into two groups by treatment Week $12 \mathrm{Hb}$ level. a Patients with diabetic nephropathy as the CKD etiology. b Patients without diabetic nephropathy as the CKD etiology. c Patients with nephrosclerosis as the CKD etiology. d Patients without nephrosclerosis as the CKD etiology. e Patients aged $<75$ years. f Patients aged $\geq 75$ years. a Overall renal survival rate by Kaplan-Meier analysis was $23.45 \%$ in the $<11 \mathrm{~g} / \mathrm{dL}$ group and $45.11 \%$ in the $\geq 11 \mathrm{~g} /$ $\mathrm{dL}$ group for patients with diabetic nephropathy as the CKD etiology. b Overall renal survival rate by Kaplan-Meier analysis was $45.33 \%$ in the $<11 \mathrm{~g} / \mathrm{dL}$ group and $55.13 \%$ in the $\geq 11 \mathrm{~g} / \mathrm{dL}$ group for patients without diabetic nephropathy as the CKD etiology. c Overall renal survival rate by Kaplan-Meier analysis was $59.69 \%$ in the $<11 \mathrm{~g} / \mathrm{dL}$ group and $69.58 \%$ in the $\geq 11 \mathrm{~g} / \mathrm{dL}$ group for patients with nephrosclerosis as the CKD etiology. d Overall renal survival rate by Kaplan-Meier analysis was $29.21 \%$ in the $<11 \mathrm{~g} / \mathrm{dL}$ group and $44.57 \%$ in the $\geq 11 \mathrm{~g} / \mathrm{dL}$ group for patients without nephrosclerosis as the CKD etiology. e Overall renal survival rate by KaplanMeier analysis was $19.85 \%$ in the $<11 \mathrm{~g} / \mathrm{dL}$ group and $42.81 \%$ in the $\geq 11 \mathrm{~g} / \mathrm{dL}$ group for patients aged $<75$ years. f Overall renal survival rate by Kaplan-Meier analysis was $54.25 \%$ in the $<11 \mathrm{~g} / \mathrm{dL}$ group and $61.70 \%$ in the $\geq 11 \mathrm{~g} / \mathrm{dL}$ group for patients aged $\geq 75$ years. Week 0: C.E.R.A. treatment week 12 was used as the landmark time (start time). Hb, Hemoglobin; N, number of patients

patients. Furthermore, the assessment of responsiveness to ESAs in terms of ERI was not performed. Finally, the use of landmark analysis starting at treatment Week 12 meant that 1750 patients in the safety analysis set were excluded. Data on week $12 \mathrm{Hb}$ levels were missing for 1568 of these patients. Once monthly measurement of $\mathrm{Hb}$ levels was specified in advance in the protocol, but, because this was an observational study under actual-use conditions, monthly patient hospital visits, and laboratory testing could not be enforced. Nevertheless, there is thought to be no selection bias, because (1) there were no noteworthy clinical differences in patient baseline characteristics between the 4601 safety analysis set patients and the 2851 landmark analysis set patients and (2) the incidence of renal events in the landmark analysis set was roughly the same as previously reported incidences of renal events $[13,18]$.

The strengths of the study are as follows. First, the results are directly applicable into real-world clinical practice, because a large number of patients and institutions participated, reflecting the clinical situation in Japan. Second, despite being an observational study, adequate patient information and test data were obtained, and reliable analysis was performed. Finally, the study complied with the Japanese Ministry of Health, Labour and Welfare's GPSP standards, and there was thorough adverse event surveillance. In the real-world clinical setting, we consider it desirable to set $\mathrm{Hb}$ treatment targets based on the patient's condition, with reference to the results of this study and based on recommendations in the 2015 JSDT "Guidelines for Renal Anemia in Chronic Kidney Disease" [14].

\section{Conclusion}

This study investigated the relationship between the achieved $\mathrm{Hb}$ level and renal outcome when renal anemia is treated with C.E.R.A. in Japanese NDCKD patients in the actual clinical setting.

The results suggest that renal outcomes are better when the achieved $\mathrm{Hb}$ level is $\geq 11 \mathrm{~g} / \mathrm{dL}$ than when it is $<11 \mathrm{~g} /$ $\mathrm{dL}$, but an RCT is needed to verify these results. 


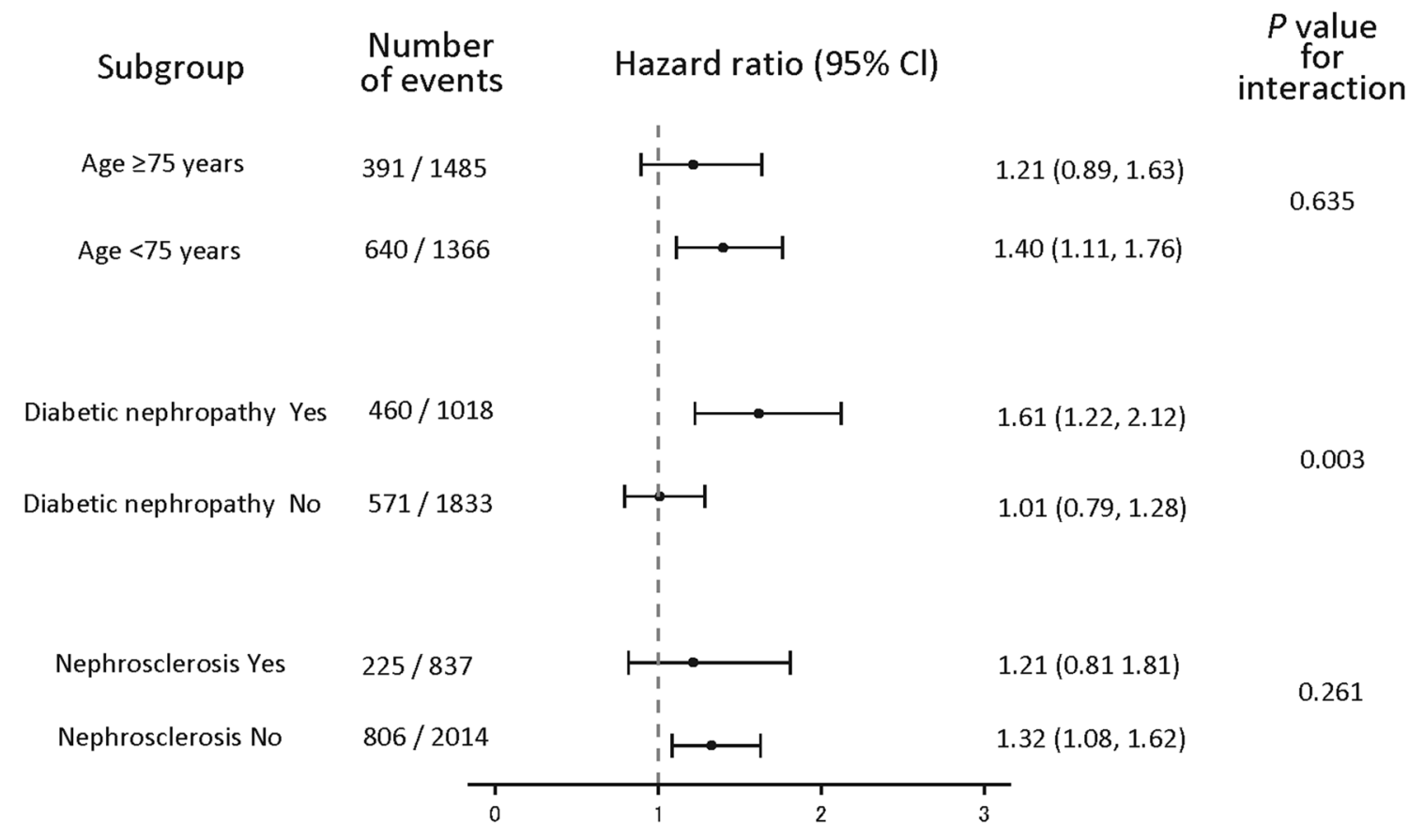

Fig. 6 Subgroup analyses by age and CKD etiology. Hazard ratios and 95\% CI were adjusted for multivariate Cox regression analysis. Hazard ratio of group with Week $12 \mathrm{Hb}$ level $<11 \mathrm{~g} / \mathrm{dL}$ when hazard ratio of group with Week $12 \mathrm{Hb}$ level $\geq 11 \mathrm{~g} / \mathrm{dL}$ is 1 . CI, confidence interval

Table 3 Adverse drug reactions

\begin{tabular}{|c|c|c|c|c|c|c|c|c|}
\hline \multirow[t]{3}{*}{ Category } & \multirow{2}{*}{\multicolumn{2}{|c|}{$\begin{array}{l}\text { Safety analysis } \\
\text { set }(N=4601)\end{array}$}} & \multirow{2}{*}{\multicolumn{2}{|c|}{$\begin{array}{l}\text { Landmark } \\
\text { analysis set }(N= \\
2851)\end{array}$}} & \multicolumn{4}{|c|}{ Week $12 \mathrm{Hb}$} \\
\hline & & & & & \multicolumn{2}{|c|}{$<11(N=1793)$} & \multicolumn{2}{|c|}{$\begin{array}{l}\geq 11(N= \\
1058)\end{array}$} \\
\hline & Serious & Total & Serious & Total & Serious & Total & Serious & Total \\
\hline No. of patients with ADRs & 98 & 195 & 61 & 122 & 39 & 77 & 22 & 45 \\
\hline No. of occurrences of ADRs & 124 & 238 & 77 & 147 & 49 & 92 & 28 & 55 \\
\hline ADR incidence $(\%)$ & 2.12 & 4.23 & 2.13 & 4.27 & 2.17 & 4.29 & 2.07 & 4.25 \\
\hline
\end{tabular}

No., number of patiens; ADR, adverse drug reaction; Hb, Hemoglobin

Acknowledgements The authors would like to express our deepest gratitude to the patients, in addition to the investigators and staff at the study sites (presented in the Online appendix) for their contribution to the study. This study was sponsored by Chugai Pharmaceutical, Co., Ltd. (Tokyo, Japan).

\section{Compliance with ethical standards}

Conflict of interest Employment/Leadership position/Advisory role: YO (Statcom), MKu (Chugai), MKi (Chugai), HK (Chugai). Stock ownership or options: YO (Statcom). Honoraria: TH (Kyowa Hakko Kirin, Chugai), YU (Chugai), YO (Chugai, Daiicchi-Sankyo, Sanofi). Research funding: YO (Eisai, Yakult Honsya).

Ethical approval A third-party central institutional review board (AiResearch Co., Ltd.) approved the study protocol (Approval no. 70), and the study was conducted in accordance with the Declaration of Helsinki and Japanese Ministry of Health, Labour and Welfare regulations for post-marketing surveillance.
Registration of clinical trials This study was also registered in advance at the University Hospital Medical Information Network Center (ID: UMIN000008568).

Informed consent This study was conducted in compliance with Good Post-Marketing Study Practice (GPSP) standards defined by the Japanese Ministry of Health, Labour and Welfare, which does not require informed consent. A third-party central institutional review board (AiResearch Co., Ltd.) approved the ethical integrity of the study protocol.

Open Access This article is distributed under the terms of the Creative Commons Attribution 4.0 International License (http://creativeco mmons.org/licenses/by/4.0/), which permits unrestricted use, distribution, and reproduction in any medium, provided you give appropriate credit to the original author(s) and the source, provide a link to the Creative Commons license, and indicate if changes were made. 


\section{References}

1. McClellan W, Aronoff SL, Bolton WK, et al. The prevalence of anemia in patients with chronic kidney disease. Curr Med Res Opin. 2004;20:1501-10.

2. Portolés J, Gorriz JL, Rubio E, et al. The development of anemia is associated to poor prognosis in NKF/KDOQI stage 3 chronic kidney disease. BMC Nephrol. 2013;14:2.

3. Gouva C, Nikolopoulos P, Ioannidis JP, Siamopoulos KC. Treating anemia early in renal failure patients slows the decline of renal function: a randomized controlled trial. Kidney Int. 2004;66:753-60.

4. Akizawa $\mathrm{T}$, Tsubakihara $\mathrm{Y}$, Hirakata $\mathrm{H}$, et al. A prospective observational study of early intervention with erythropoietin therapy and renal survival in non-dialysis chronic kidney disease patients with anemia: JET-STREAM Study. Clin Exp Nephrol. 2016;20:885-95.

5. Singh AK, Szczech L, Tang KL, et al. Correction of anemia with epoetin alfa in chronic kidney disease. N Engl J Med. 2006;355:2085-98.

6. Drueke TB, Locatelli F, Clyne N, et al. Normalization of hemoglobin level in patients with chronic kidney disease and anemia. N Engl J Med. 2006;355:2071-84.

7. Pfeffer MA, Burdmann EA, Chen CY, et al. A trial of darbepoetin alfa in type 2 diabetes and chronic kidney disease. N Engl J Med. 2009;361:2019-32.

8. Akizawa T, Pisoni RL, Akiba T, et al. Japanese haemodialysis anaemia management practices and outcomes (19992006): results from the DOPPS. Nephrol Dial Transpl. 2008;23:3643-53.

9. Kidney Disease: Improving Global Outcomes (KDIGO) CKD Work Group. KDIGO 2012 clinical practice guideline for anemia in chronic kidney disease. Kidney Int. 2012;2(suppl):279-335.
10. Szczech LA, Barnhart HX, Inrig JK, et al. Secondary analysis of the CHOIR trial epoetin- $\alpha$ dose and achieved hemoglobin outcomes. Kidney Int. 2008;74:791-8.

11. McCullough PA, Barnhart HX, Inrig J, et al. Cardiovascular toxicity of epoetin-alfa in patients with chronic kidney disease. Am J Nephrol. 2013;37:549-58.

12. Solomon SD, Uno H, Lewis EF, et al. Erythropoietic response and outcomes in kidney disease and type 2 diabetes. $\mathrm{N}$ Engl $\mathrm{J}$ Med. 2010;363:1146-55.

13. Tsubakihara Y, Gejyo F, Nishi S, et al. High target hemoglobin with erythropoiesis-stimulating agents has advantages in the renal function of non-dialysis chronic kidney disease patients. Ther Apher Dial. 2012;16:529-40.

14. Yamamoto H, Nishi S, Tomo T, et al. Japanese society for dialysis therapy: guidelines for renal anemia in chronic kidney disease. Renal Replacement Thrapy 2015. 2017;3:36.

15. Yuzawa Y, Horita S, Suzuki Y, et al. Effect of hemoglobin (Hb) maintenance of subcutaneous (SC) or intravenous (IV) C.E.R.A. (continuous erythropoietin receptor actvator) in chronic kidney disease (CKD) patients not on dialysis. Jpn Pharmacol Ther. 2011;39:s55-s68.

16. Nicola L, Conte G, Chiodini P, et al. Stability of target hemoglobin levels during the first year of epoetin treatment in patients with chronic kidney disease. Clin J Am Soc Nephrol. 2007;2:938-46.

17. Tsuruya K, Uemura Y, Hirakata H, et al. Association between responsiveness to methoxy polyethylene glycol-epoetin beta and renal survival in patients with non-dialysis-dependent chronic kidney disease: a pooled analysis of individual patient-level data from clinical trials. Nephrology. 2017;22:769-75.

18. Kuriyama S, Tomonari H, Yoshida H, et al. Reversal of anemia by erythropoietin therapy retards the progression chronic renal failure, especially in nondiabetic patients. Nephron. 1997;77:176-85. 